\title{
Experimental investigation into laser ranging with sub-ns laser pulses
}

\author{
M. Hintikka and J. Kostamovaara, Senior Member, IEEE
}

\begin{abstract}
We present a high precision time-of-flight (TOF) laser radar system based on energetic $(\sim 0.6 \mathrm{~nJ})$ sub-ns laser pulses produced with a semiconductor laser diode. The proposed device has a single-shot precision of $<5 \mathrm{~mm}$ at an SNR of 10 and a maximum walk error $<500 \mathrm{ps}$ in the dynamic range $1: 250$. Sub-mm precision can be achieved in ranging by means of averaging. The proposed laser radar can be used for monitoring tiny vibrations in distant targets, for example.
\end{abstract}

Index Terms-Laser radar, laser rangefinding, optical receivers, optical sensors.

\section{INTRODUCTION}

$\mathrm{A}$ pulsed time-of-flight laser radar operates by measuring the transit time of a short laser pulse sent to the target and scattered back to the receiver. The distance from the target can then be calculated based on the known velocity of light [1]. The precision of laser radars is improving constantly, and simplicity, cost and high measurement speed (since the target may be moving) are important factors that have paved the way for the use of TOF techniques in industrial applications [2-8]. The construction of a typical pulsed TOF laser radar system is shown in Fig. 1.

Pulsed TOF laser radars typically use high energ y pulses with lengths of $3-5 \mathrm{~ns}$, allowing a single-shot precision of a few centimetres [9]. This precision can be improved by averaging multiple single measurements, but of course at the expense of increased measurement time. For example, if a $10 \mathrm{kHz}$ pulsing frequency is used (typical of high power avalanche-type pulsing devices) the averaging of 100 measurements takes $10 \mathrm{~ms}$. The main factors limiting the precision are detection noise and the timing walk (walk error) caused by variation in the amplitude of the received echo pulse [9]. Due to the relatively wide laser pulse, the walk error needs to be compensated for in distance measurement applications where high accuracy is needed. However, in measurements where small changes in distance are to be detected on top of a long base distance, e.g. where measurements are affected by tiny vibration, the walk error compensation is not crucial, since the variation in the amplitude of the received pulse is small.

When sub-cm single-shot precision is aimed at, the jitter in the timing detection should be minimized. It is well known that

This paragraph of the first footnote will contain the date on which you submitted your paper for review. This work was supported by the Academy of Finland. jitter is proportional to the ratio of the receiver noise to the slew rate of the timing signal [10]. The rise time of the laser pulse in typical radars using $3-5 \mathrm{~ns}$ pulses is of the order of a few ns, but if the laser diode is driven into the gain switching mode, a significantly shorter pulse $(\sim 100 \mathrm{ps})$ with a lower energy level $(<0.1 \mathrm{~nJ})$ can be produced $[11,12]$. It has been shown recently, however, that it is possible to markedly enhance the gain switching by increasing the equivalent spot size (d/gamma) of the laser diode. As a result, laser pulses with an energ y of $>1 \mathrm{~nJ}$ $(10 \mathrm{~W})$ and length of $\sim 100$ ps were produced with pulse current drive parameters of $\sim 10 \mathrm{~A} / 1 \mathrm{~ns} / 100 \mathrm{kHz}[13,14]$.

The goal of the present pulsed TOF study was to use this high-speed sub-ns transmitter to achieve sub-mm precision when measuring distances from passive targets. The interest lay in trying to detect small distance variations (e.g. vibrations in a machine) from a distance away. On the other hand, the short laser pulse width (= short probe length) enables better resolve double echoes which are produced when part of the laser beam hits a second, more distant target in addition to the primary one. An Avalanche Photo detector (APD) is typically used as the detector component in laser range-finder applications, due to its internal gain [15]. A silicon-based CMOS APD device would offer low cost, fast response and the possibility of integrating the detector with the receiver and other electronics on the same chip, but its responsivity is markedly lower than that of specialized commercial photo-detectors [16]. The measurements in this work, were made mainly using a discrete APD, but a CMOS-based APD chip was also tested for asses its usability in pulsed TOF ranging.

The paper is organized as follows: Section II describes the design principles and background theory, after which the construction of a laser radar device is explained in section III. Section IV presents the measurement results and an evaluation of the performance of the range finder. Conclusions are drawn in section $\mathrm{V}$.

\section{DESIGN PRINCIPLES AND SUB-NS DETECTION}

Typical pulsed TOF laser radars intended for industrial applications use a laser diode transmitter producing high power $(>10 \mathrm{~W})$ pulses with a typical length of $<5 \mathrm{~ns}$ (FWHM). The pulse length is limited to the nanosecond range due to the limitations of high-current (peak current $>10 \mathrm{~A}$ ), high speed drivers $[17,18]$. However, a number of special gain-switched laser constructions have been suggested which can produce energetic sub-ns range pulses $[19,20]$. In particular, 'enhanced

The authors are with the Circuits and Systems research group, University of Oulu, 90014 University of Oulu, Finland (e-mail: mikko.hintikka@oulu.fi; juha.kostamovaara@oulu.fi ). 
gain switching'-based constructions are capable of producing laser pulses with an energy level of $\sim \mathrm{nJ}$ and a pulse width of $\sim 100$ ps $[13,14,20]$.

The laser diode transmitter used here had a MOSFET current driver and a quantum well GaAs/GaAlAs laser diode working in the enhanced gain switching regime [21]. This produces optical pulses with a peak power of $6 \mathrm{~W}$ and a pulse width of $\sim 100$ ps. A discrete APD is used as the photo-detector of the laser radar because of the high sensitivity attributable to its internal gain. The sensitivity is reduced with fast pulsing due to the limited bandwidth, as shown in [16], but it was still measured to be about $10 \mathrm{~A} / \mathrm{W}$. When detecting sub-ns pulses, the bandwidth of the receiver should be matched accordingly. For a pulse width of $100 \mathrm{ps}$ (FWHM), for example, a bandwidth of $\sim 3.5 \mathrm{GHz}(\mathrm{BW}=0.35 / \mathrm{tr})$ would be needed. The limited speed of the APD (width of the impulse response $~ 300 \mathrm{ps}$ ) nevertheless restricts the bandwidth requirement of the receiver to the $\mathrm{GHz}$ range. In this work, the bandwidth of the receiver channel was set to $\sim 700 \mathrm{MHz}$, being limited by the technology used in the proposed receiver configuration. Note, however, that even though the bandwidth is lower than optimum, the effect on the jitter is only minor, since a higher bandwidth would increase the noise as well. The responsivity of a CMOS APD is low compared with a discrete one, but due to its speed and integration possibility, this is an interesting detector option for a laser range finder using sub-ns pulses. For this reason, even though a commercial discrete APD (AD100-8 SMD from First Sensor) was used in the majority of the measurements, the usability of a CMOS APD was also tested by replacing the discrete APD with a standalone CMOS APD chip (realized in $0.35 \mu \mathrm{m}$ CMOS technology) for some measurements.

The radar equation, giving an estimate for the received power, can be written for a non-cooperative (Lambertian) target as

$$
P(Z)=\frac{A_{r}}{\pi \cdot Z^{2}} \cdot P_{0} \cdot \tau \cdot \varepsilon
$$

where $P(Z)$ is the power of the receiver aperture as a function of the distance $\mathrm{Z}, P_{0}$ is the optical power of the transmitter, $A_{r}$ is the area of the receiver optics, $\varepsilon$ is the reflectance of the diffuse target and $\tau$ is the efficiency of the optics [22]. The radar equation enables one to optimize the performance of the system and to seek the optimum balance in the design parameters. This will enable estimation of the signal current used in detection, so that the signal-to-noise ratio and valid distance measurement range can be calculated. For example, the responsivity of the APD and the input-referred noise of the TIA obtained here for the receiver are $10 \mathrm{~A} / \mathrm{W}$ and $450 \mathrm{nA}(\mathrm{rms})$, respectively, meaning that primary signal current of about $450 \mathrm{nA}$ in the detector will give an SNR of 10 for the receiver (where SNR is defined as the ratio of the peak signal current to the inputreferred total noise current of the preamplifier). From (1) we can calculate that for a typical $6 \mathrm{~W}$ pulse from the abovementioned laser diode transmitter, a receiver aperture of $20 \mathrm{~mm}$, optics efficiency of 0.7 and a target having $\varepsilon=0.1$, the maximum achievable distance measurement range is $\sim 10 \mathrm{~m}$.

As already mentioned above, jitter is proportional to the ratio of the receiver noise to the slew rate of the timing signal. [10]:

$$
\sigma_{\text {jitter }} \approx \frac{\sigma_{\text {noise }}}{(\partial v / \partial t)} \approx \frac{t_{r}}{S N R} \text { and } \sigma_{R} \approx \frac{t_{r}}{S N R} \times \frac{c}{2} .
$$

Thus, as the rise time of the pulse decreases, the jitter will decrease as well if the SNR remains the same. The jitter directly affects the single-shot range precision of the laser radar $\left(\sigma_{R}\right)$.

There can be wide variations in the power of the received echo (the amplitude of the APD response) in TOF-based laser radars, and this causes the shift for the detection moment of the pulse resulting a systematic error. This is usually called timing walk [9]. Several techniques for compensating for the walk error have been presented, see [23-27] and the references therein, but it is quite obvious that the inherent walk error will be lower with shorter laser pulses, as illustrated in Fig. 2.

One challenge in TOF-based laser ranging is the change in the shape of the echo according to the orientation of the target. In extreme cases even multiple echoes can be detected, as is shown in Fig. 3, where the laser spot is split in half at a sharp edge on the target. In the case illustrated in Fig. 3 the pulse shape grows wide depending on the depth of the step in the target. When the step is deep enough, two different pulses reflect from the target (each half of the spot). Thus, it depends strongly on the width of the pulse as to how short the distance differences are that can be resolved with the radar. The situation shown in Fig. 3 is one in which the edge can be identified with a sub-ns pulse, and it also illustrates the situation with a wider pulse when identification is not possible.

\section{CONSTRUCTION OF THE LASER RADAR}

The laser radar constructed here consists of a receiver APD detector (discrete: diameter $100 \mu \mathrm{m}, \mathrm{CMOS}: 20 \mu \mathrm{m}$ x $40 \mu \mathrm{m}$ ), paraxial optics, a laser transmitter and the receiver electronics, including the receiver channel, time-to-digital converter (TDC) and controlling FPGA board. A block diagram of the system device is presented in Fig. 4.

The laser radar has a transmitter using a MOSFET-based driver presented in [21]. This is a compact pulser emitting $0.6 \mathrm{~nJ} / 100 \mathrm{ps}$ pulses up to a pulsing frequency of over $100 \mathrm{kHz}$. The high pulsing frequency is achieved using a MOSFET transistor in the pulsing device instead of an avalanche transistor with a high on-voltage. The laser diode is based on a quantum well structure with a cavity length of $1.5 \mathrm{~mm}$ and an emitting stripe width of $30 \mu \mathrm{m}$ [20]. The schematics of the driver are presented in Fig. 5 and a photograph of the laser transmitter is shown in Fig. 6. The light emitted by the laser diode was focused on the fibre carrying light to the transmitter optics.

The receiver uses paraxial optics in which the focal lengths of the transmitter and receiver optics are $30 \mathrm{~mm}$ and $20 \mathrm{~mm}$, respectively. The receiver aperture is $20 \mathrm{~mm}$. The diameter of the optical fibre used in focusing the transmitter was $50 \mu \mathrm{m}$, which leads to a spot size of $\sim 3 \mathrm{~mm}$ at a distance of $2 \mathrm{~m}$. The optics used here are shown in Fig. 7.

The receiver channel circuit was fabricated in a $0.18 \mu \mathrm{m}$ HVCMOS technology and included a transimpedance preamplifier, a post-amplifier and a timing comparator which 
discriminates the timing signals from the leading and the trailing edges, giving two stop pulses (Stop 1 for the leading edge of the pulse and Stop 2 for the trailing edge) [28]. Both edges are discriminated, which in principle also allows walk error compensation. The bandwidth of the receiver is $\sim 700$ $\mathrm{MHz}$ and the equivalent transimpedance $\sim 25 \mathrm{k} \Omega$. The input referred noise current is approximately $450 \mathrm{nA}$ with a discrete APD at the input [28]. The transimpedance amplifier and the comparator use a $1.8 \mathrm{~V}$ supply voltage, but thanks to the high voltage (HV) design, the internal level shifters giving a 2.5 to $5 \mathrm{~V}$ output are located in the chip and can thus be used with a time-to-digital converter (TDC) using a supply voltage of $3.3 \mathrm{~V}$. A photograph of the receiver channel chip is shown in Fig. 8.

The time interval measurements circuit, as presented in detail in [29], is a multichannel TDC implemented in a $0.35 \mu \mathrm{m}$ technology and giving a timing resolution $<10 \mathrm{ps}$. The supply voltage of the TDC is $3.3 \mathrm{~V}$ and a block diagram is shown in Fig. 9. The data obtained from the TDC (18 bytes per measurement) are transferred to an FPGA interface by 8 parallel lines. The FPGA board also resets the comparators after reading the TDC data, so that the laser radar device is ready for the next measurement. The FPGA board is controlled by the computer's test software.

\section{MEASUREMENTS}

The measurements were carried out using a sheet of white paper with diffuse reflectance of $\varepsilon \sim 1$ as the reference target. The shape of the laser pulse as measured with a high-speed optical probe $(\sim 25 \mathrm{GHz})$ is shown in Fig. 10, and the response of the receiver channel is depicted in Fig. 11. The pulse in Fig. 11 was probed from the output buffer of the receiver when measuring a distance of $6 \mathrm{~m}$. The input-referred detection noise was $450 \mathrm{nA}$ so that the noise level at the output of the receiver was $11.5 \mathrm{mV}$ when the transimpedance of the channel was $25 \mathrm{k} \Omega$. The pulse of amplitude $400 \mathrm{mV}$ in Fig. 11 corresponds to an SNR of 36. The measurement is the average of 1,024 measurements, so that the noise level is attenuated. There is, however, a periodic crosstalk bounce after the pulse which originates from the comparator reset and does not affect the results.

The single-shot jitter of the rising edge of the detected pulse was measured by sweeping the amplitude of the laser pulse with a variable neutral density filter. The jitter recorded with three detection thresholds can be seen in Fig. 12. The threshold levels are shown as SNR (blue: $80 \mathrm{mV}, \mathrm{SRN}=7$, red: $126 \mathrm{mV}, \mathrm{SNR}$ $=11$ and green: $195 \mathrm{mV} \mathrm{SNR}=17$ ). The results show an improvement in the jitter as a function of signal amplitude (and thus SNR), and a jitter saturation towards the bottom value restricted by the jitter of the TDC (10 ps). The y axis on the right shows the corresponding range jitter. Note also that the jitter at a low signal SNR is somewhat higher for a higher threshold (e.g. $V_{\text {th }}$ is equivalent to $\mathrm{SNR}=17$ ), than for lower thresholds, on account of the decreasing signal slew rate near the peak of the signal pulse.

The walk error was measured by sweeping the amplitude of the laser pulse from $\mathrm{SNR}=10$ to $\mathrm{SNR}=2,500$ (setting the comparator threshold at SNR =7). The high SNR of 2,500 corresponds to a signal current of $\sim 1 \mathrm{~mA}$, which is well beyond the linear range of the preamplifier. Note, however, that this does not destroy the leading edge timing discrimination. Also, external cross-coupled Schottky diodes are used to limit the input current to the preamplifier and to speed up its recovery from the transient. The measured walk error can be seen in Fig. 13. It is measured by sweeping the echo amplitude trough the dynamic range of 1:250 and the Fig. 13 shows the shift of the timing moment as a function of echo amplitude. Timing walk is a notable source of error in TOF-based laser ranging when measuring distances within a wide dynamic range of echo pulses, and with the transceiver presented here it is $\sim 500 \mathrm{ps}$ ( $7.5 \mathrm{~cm}$ in distance) with a linear dynamic range of the received echo amplitudes of 1:250. This error is markedly lower than that typically achieved in a pulsed TOF laser radar using a conventional ns-scale pulse width, e.g. $2.5 \mathrm{~ns}$ walk error with a $\sim 3 \mathrm{~ns}$ laser pulse [1, 4] or $\sim 1.7 \mathrm{~ns}$ with an ns pulse [23]. Although the walk error is usually compensated for, it is of less importance in this particular case, where the vibration of the surface is mainly targeted and the echo amplitude is high and relatively constant.

The precision of the system with respect to distance measurement is demonstrated in Fig. 14. The target, with a steplike profile ( $2 \mathrm{~mm}$ in height), was moved vertically (total movement $2.5 \mathrm{~cm}$ ) with regard to the optical axis of the laser radar while the radar was continuously measuring the distance. The diameter of the laser spot at a distance of $2 \mathrm{~m}$ was around $3 \mathrm{~mm}$. Single-shot measurement results were recorded at a pulsing rate of $24 \mathrm{kHz}$. Fig. 14 a) shows the measurements without averaging (single-shot) and b), c) and d) the averaged results of 10,100 and 1,000 single-shot measurements, respectively. It can be seen that sub-mm precision can easily be achieved for a high signal level (SNR 2,000) by means of averaging. Averaging increases the measurement time and, as $24 \mathrm{kHz}$ was used as the pulsing frequency, 100 measurements correspond to $\sim 4 \mathrm{~ms}$ in measurement time. On the other hand, the transmitter enables one to use a pulsing rate of up to 100 $\mathrm{kHz}$, which corresponds to a measurement time of $1 \mathrm{~ms}$. In conclusion, sub-mm precision can be achieved for relatively rapidly vibrating surfaces. The vibrating surface was simulated by recording the distance of an eccentric round target rolled by an electric motor at a base distance of $2 \mathrm{~m}$. The vibration frequency and amplitude were $10 \mathrm{~Hz}$ and $\sim 1.5 \mathrm{~mm}$, respectively. The results for single-shot measurement and for the averaging of 10,100, and 1,000 successive results are shown in Fig. 15 a), b), c) and d).

The pulse probed from the output buffer of the receiver when measuring the distance to a white paper target $1 \mathrm{~m}$ away when the discrete APD detector was replaced with the standalone CMOS APD is to be seen in Fig. 16. The detector details are presented in $[16,30]$. The reverse bias voltage was set at $18.9 \mathrm{~V}$ (close to the breakdown point) in order to maximize the response (but without any notable rise in noise level). The measured pulse response shown in Fig. 16 demonstrates a peak amplitude of $\sim 200 \mathrm{mV}$, and a responsivity of $\sim 0.3 \mathrm{~A} / \mathrm{W}$ (18.9 V bias) for the CMOS APD can be calculated from (1). That very much lower than with the discrete APD $(\sim 10 \mathrm{~A} / \mathrm{W})$, of course, but it does allow the echo pulse to be detected. In reality, as the CMOS APD could be constructed as an on-chip 
device, the noise of the receiver channel would also be reduced due to the lower input capacitance.

The light spot (of finite size) in a TOF-based laser radar may be split when hitting the edge of the target, and thus two echo pulses could be received (see Fig. 3). When using typical pulse widths of 3-5 ns, the minimum resolvable distance that can be observed is matter of several metres, but it was obviously shorter with the sub-ns pulse and laser radar used here. The situation for two observed echo pulses is illustrated in Fig. 17. The distances of the two objects in the case shown in the figure were $4.5 \mathrm{~m}$ and $5 \mathrm{~m}$ (a step of $0.5 \mathrm{~m}$ ) and the result indicates that the objects can be resolved. The minimum resolvable distance is reached when the echo pulses exceed the zero level (or the comparator threshold) between the two pulses, which would be around $0.4 \mathrm{~m}$ with this setup.

\section{CONCLUSIONS}

The hardware (HW) of the TOF-based laser radar using sub-ns laser pulses presented here consists of a MOS-based laser diode transmitter generating $100 \mathrm{ps} 0.6 \mathrm{~nJ}$ laser pulses, receiver electronics including a full-custom $0.18 \mu \mathrm{m} \mathrm{HVCMOS}$ receiver channel, a full-custom $0.35 \mu \mathrm{m}$ CMOS TDC and an FPGAbased control board.

The walk error of the radar is $\sim 500$ ps within the dynamic range of 1:250, and the jitter of the leading edge of the detected pulse is limited by the TDC to $\sim 10 \mathrm{ps}$ at a high signal-to-noise ratio. The key result of this work is that it demonstrates sub-mm precision with a relatively short measuring time. For example, it was shown that perpendicular vibration with an amplitude of $1.5 \mathrm{~mm}$ at a frequency of $10 \mathrm{~Hz}$ can be reliably observed. The measurement speed of the current realization is limited by the pulsing rate of $24 \mathrm{kHz}$, but this is not a fundamental limit and can be increased above $100 \mathrm{kHz}$ even with a $10 \mathrm{~W}$ peak pulse power level [20, 21].

The CMOS APD tested as a detector component for the receiver can be used successfully in a pulsed time-of-flight laser radar when measuring short distances or using good reflectors as targets. This is an interesting option for a detector, since it can be integrated multiply on the same die as the receiver and the rest of the electronics.

The good jitter properties of the present laser radar are due to the high speed of its pulse, and the short pulse width gives an additional advantage of enabling the resolution of better targets that are close to each other and under the laser beam. On the other hand, the optical energy is also lower (as compared with a pulse width of 3-5 ns), limiting the maximum range. An interesting compromise would then be to use a high equivalent spot size to increase the front edge speed of the laser pulse and drive the laser diode to a quasi-steady mode with a resulting optical pulse width of $\sim 1 \mathrm{~ns}$, for example. This would give a good combination of high single-shot precision and sensitivity due to the improved signal-to-noise ratio.

For future improvements an increase in receiver bandwidth would improve the walk and jitter properties, but it would also need a TDC with better single-shot precision. Integrating the CMOS APD and the receiver electronics on the same chip would reduce the jitter due to the faster detector and lower input capacitance (wider achievable bandwidth), but only at the cost of reduced sensitivity.

\section{ACKNOWLEDGMENTS}

The authors acknowledge financial support from the Academy of Finland (Centre of Excellence in Laser Scanning Research, contract no. 272196, and contracts nos. 255359, 263705 and 251571) and the Infotech Oulu Graduate School.

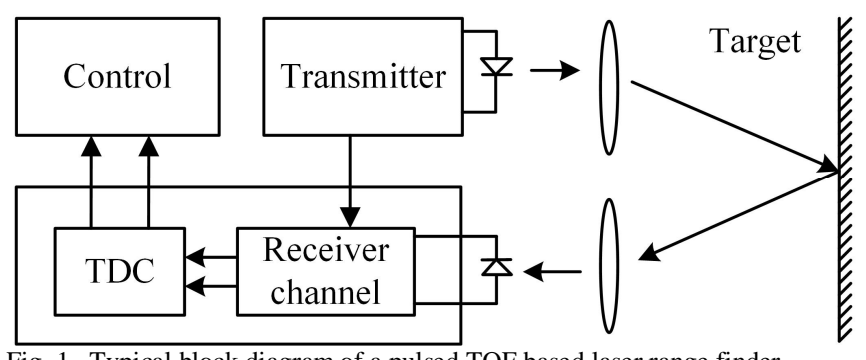

Fig. 1. Typical block diagram of a pulsed TOF based laser range finder.

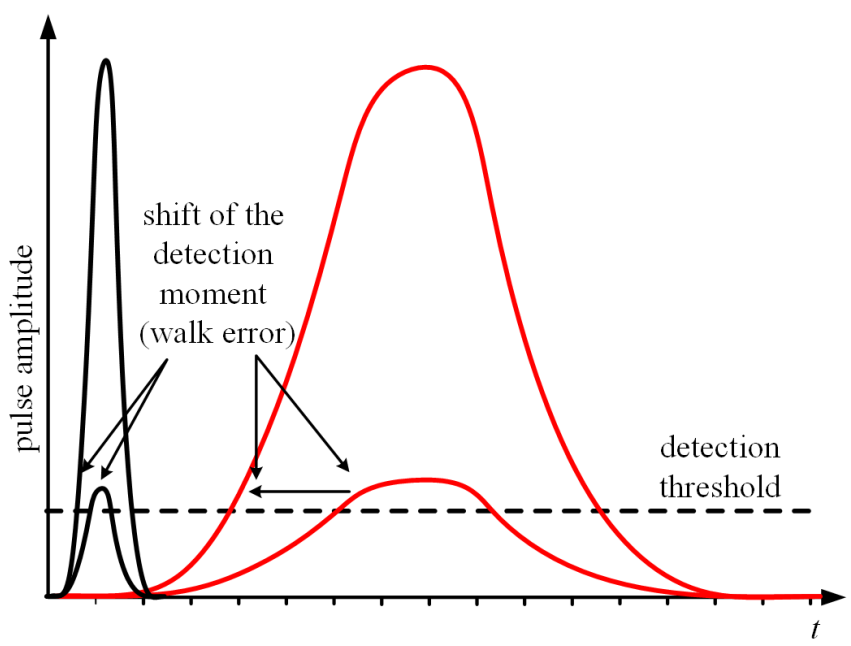

Fig. 2. Timing walk error in leading edge of the pulse.

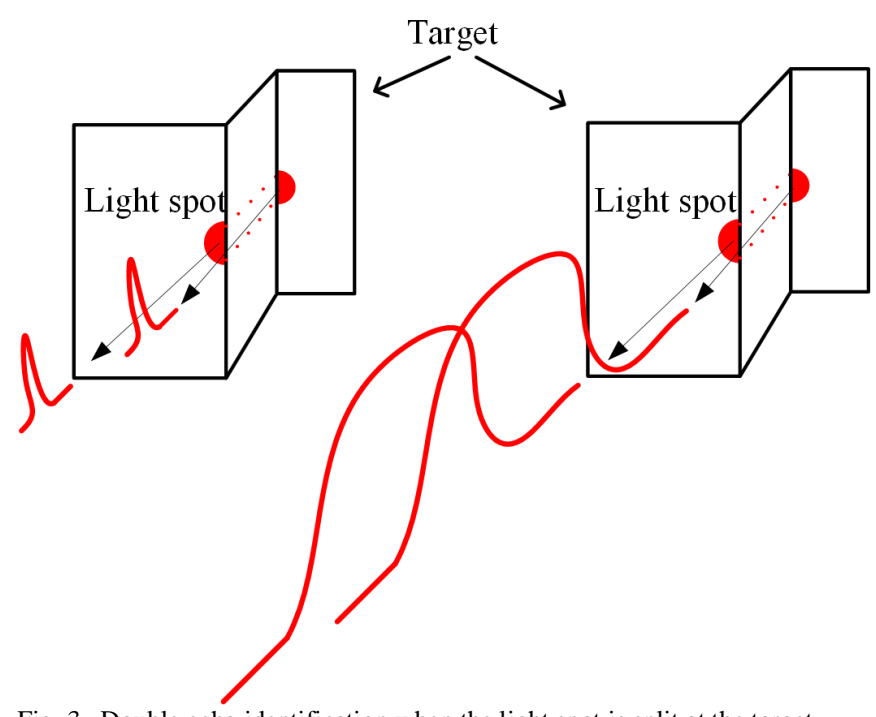

Fig. 3. Double echo identification when the light spot is split at the target. 


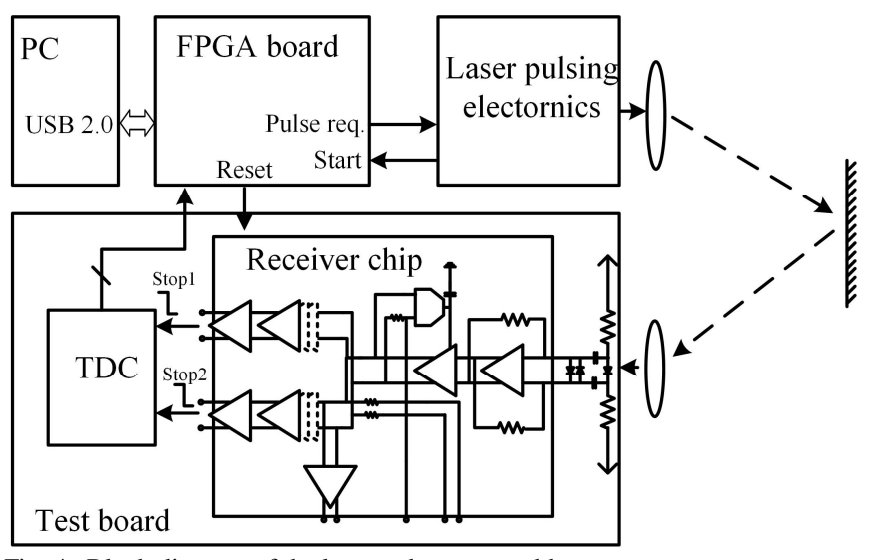

Fig. 4. Block diagram of the laser radar proposed here.
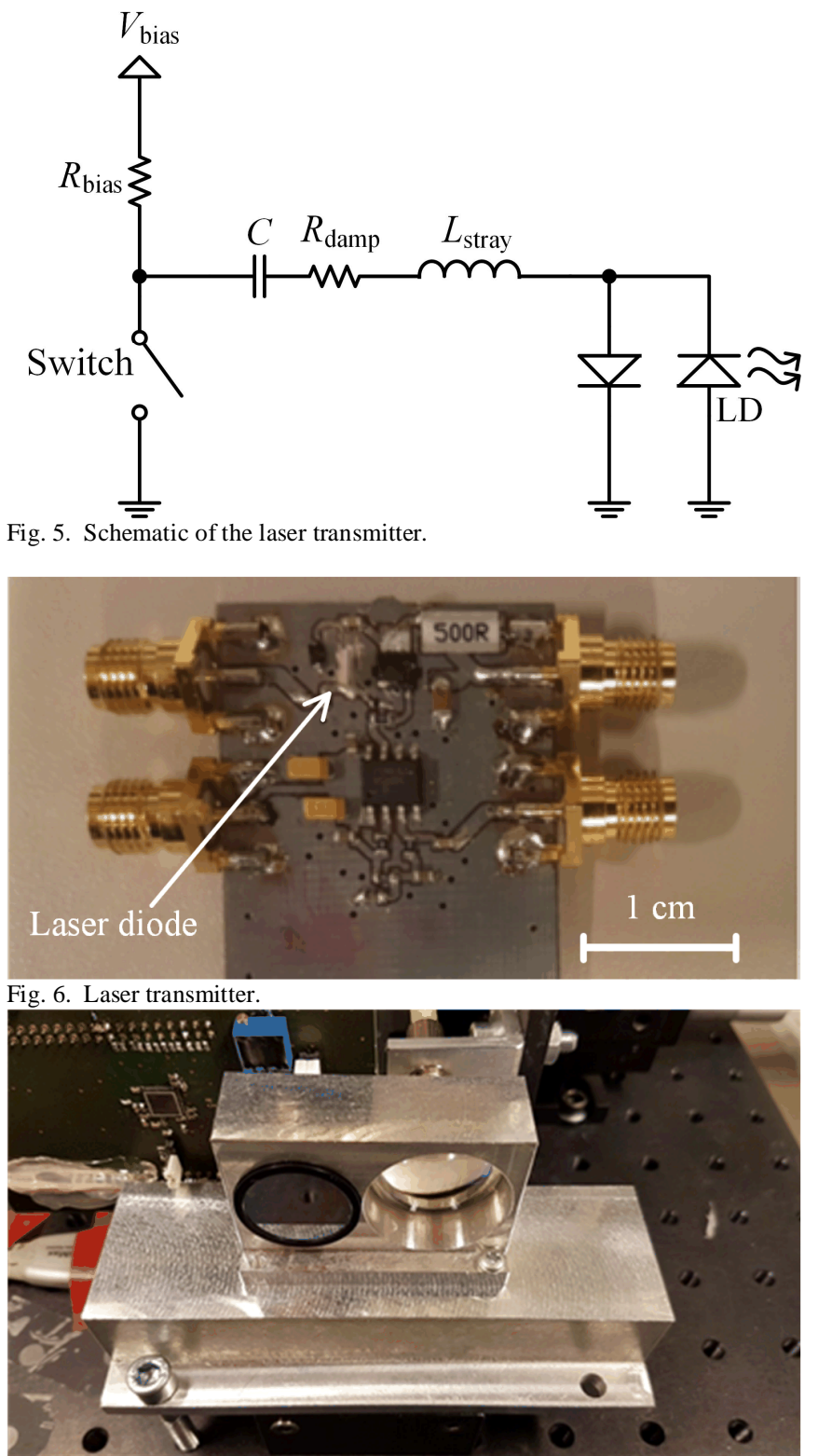

Fig. 7. Paraxial optics.

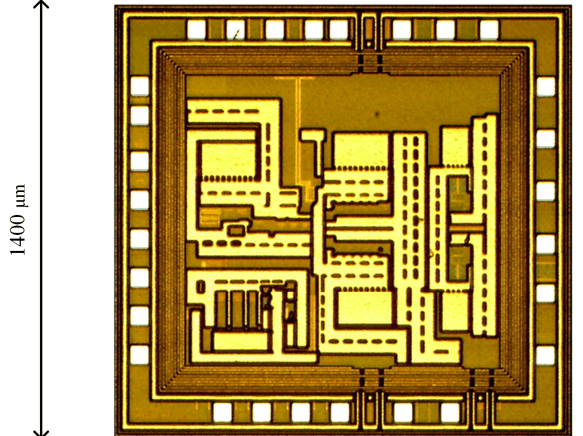

Fig. 8. Photograph of the receiver.

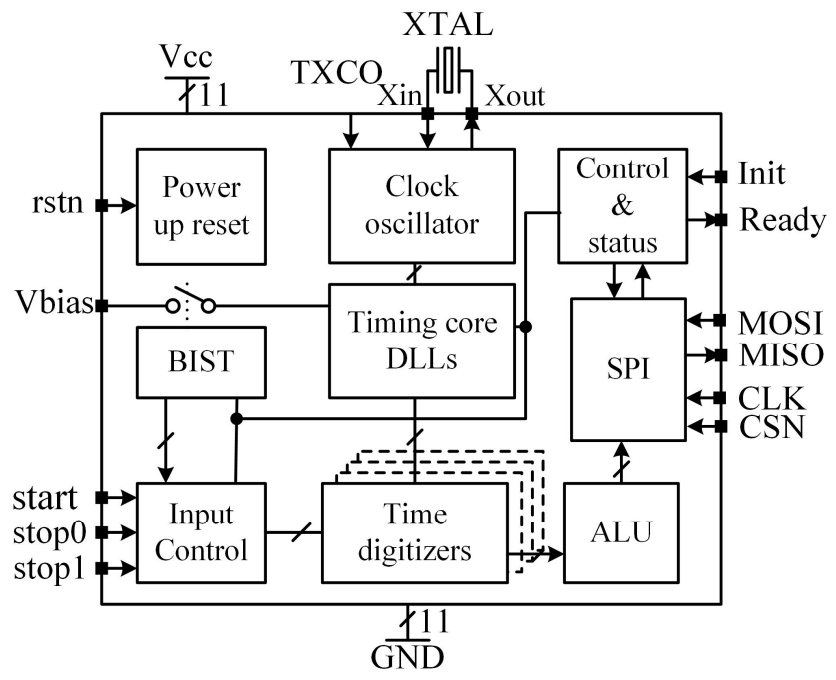

Fig. 9. Block diagram of the TDC.

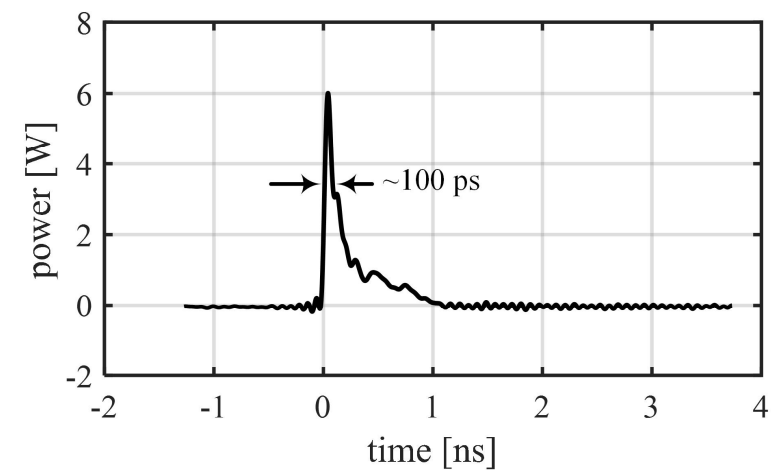

Fig. 10. Laser pulse shape probed with a high speed optical probe (New port 1434-50). 


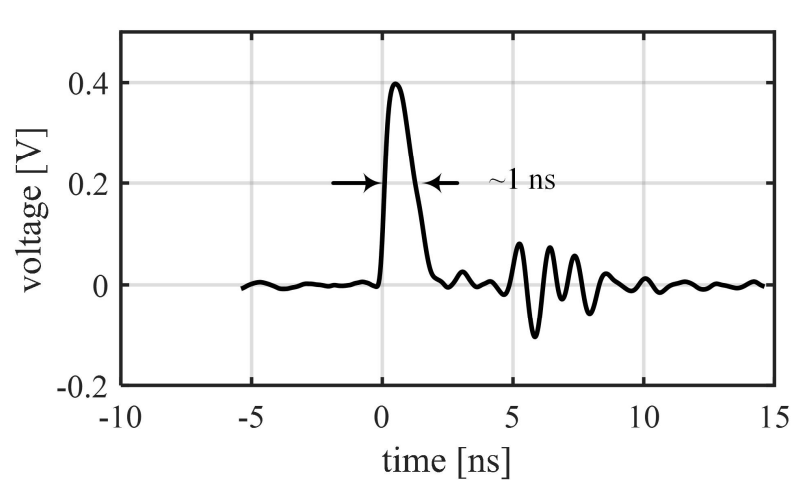

Fig. 11. Laser pulse probed from the output buffer of the receiver.

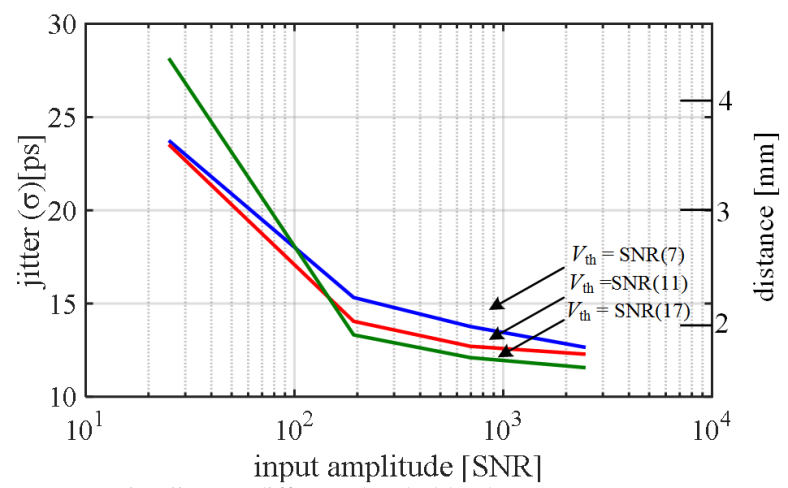

Fig. 12. Detection jitter at different threshold values.

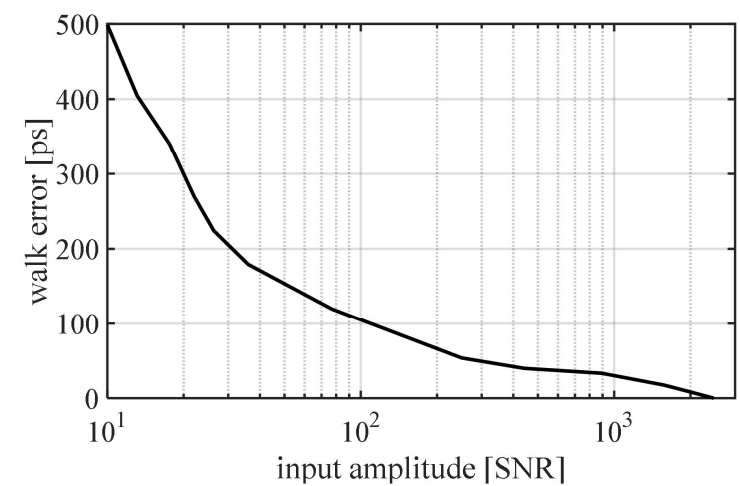

Fig. 13. Timing walk error as a function of input amplitude.
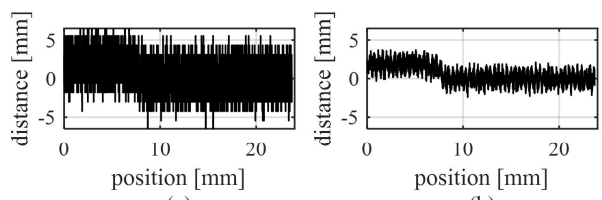

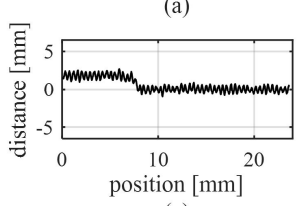

(c)

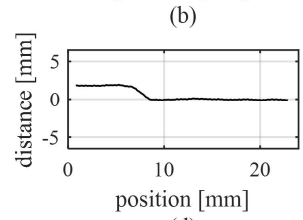

(d)

Fig. 14. A $2 \mathrm{~mm}$ step in the target profile measured with the proposed range finder. a) no averaging, b) 10 successive single-shot results averaged, c) 100 successive results averaged, d) 1,000 successive results averaged.

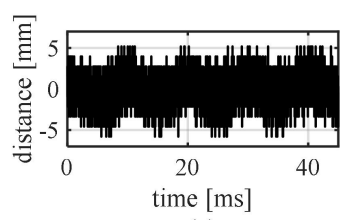

(a)

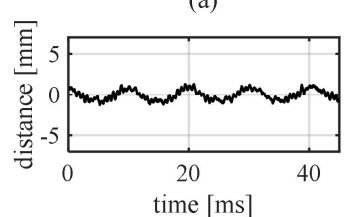

(c)

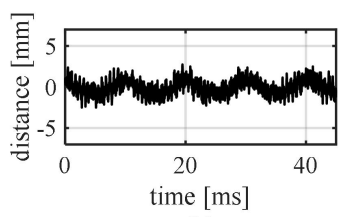

(b)

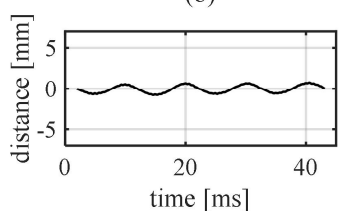

(d)
Fig. 15. $10 \mathrm{~Hz}$ vibration with an amplitude of $1.5 \mathrm{~mm}$ observed with the proposed range finder. a) no averaging, b) 10 successive single-shot results averaged, c) 100 successive results averaged, d) 1,000 successive results averaged.

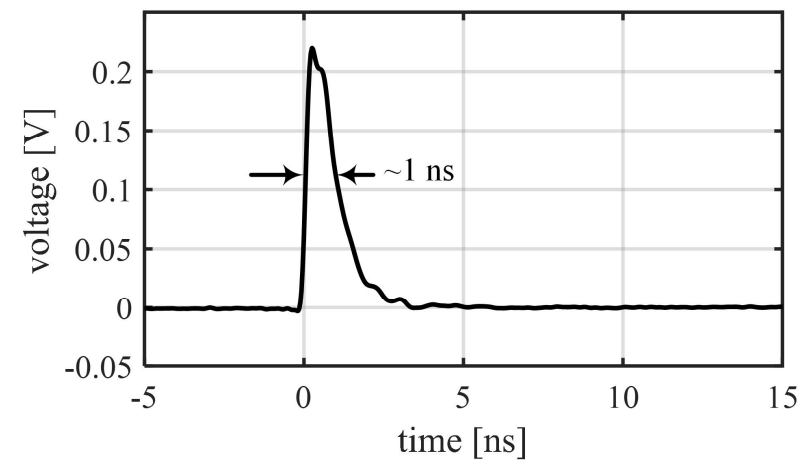

Fig. 16. A laser pulse detected with a CMOS APD and probed from the output buffer of the receiver.

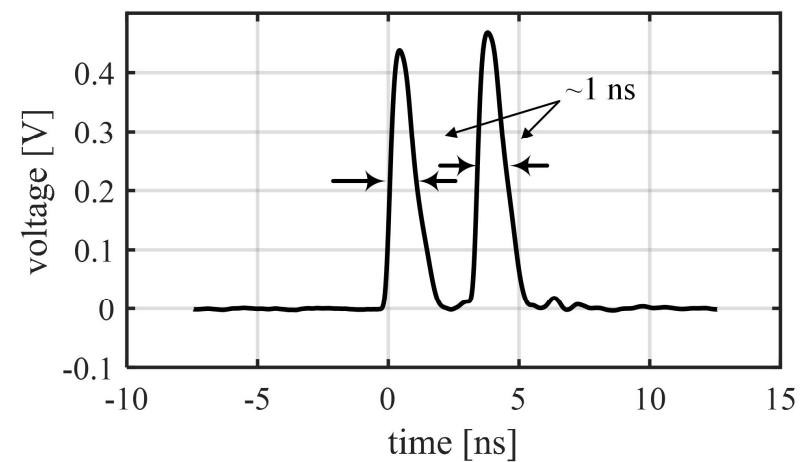

Fig. 17. Double echo probed from the output buffer of the receiver.

\section{REFERENCES}

[1] S. Donati, Electro-Optical Instrumentation: Sensing and Measuring With Lasers. Upper Saddle River, NJ, USA:

[2] R. Chen, Y. Meng, Z. Liu, J. and H. Jiang, "High precision infrared pulse laser ranging for active vehicle anti-collision application," ICEICE, Wuhan, April 2011, pp. 1404-1407.

[3] M. Lee, and S.H. Baeg, "Advanced compact 3D LIDAR using a high speed fiber coupled pulsed laser diode and a high accuracy timing discrimination readout circuit," Proc of SPIE Laser Radar Technology and Applicatons XVII, vol. 879, 8379Z, May 2012.

[4] H. -S. Cho, C. -H. Kim, and S. -G. Lee "A high-sensitivity and low-walk error LADAR receiver for military application," IEEE Trans. Circ. SystI, vol. 61, no. 10, pp. 3007-3015, Sept. 2014. 
[5] L. Viarani, D. Stoppa, L. Gonzo, M. Gottardi and A. Simoni, "A CMOS Smart pixel for active 3-D vision applications” IEEE Sensors Journal, vol 4, no. 1, Feb 2004.

[6] T. Fersch, R. Weigel and A. Koelpin, "A CDMA Modulation Technique for Automotive Time-of-Flight LiDAR systems,” IEEE Sensors Journal, vol. 17, no. 11, June 2017.

[7] A. Spickermann, D. Durini, A. Süss, W. Ulfig, W. Brockherde, B. J Hosticka, S. Schwope and A. Grabmaier "CMOS 3D image sensor based on pulse modulated time-of-flight principle and intrinsic lateral drift-field photodiode pixels", IEEE (ESSCIRC), Oct 2011, DOI: 10.1109/ESSCIRC.2011.6044927.

[8] S. Jahromi, J-P Jansson and J. Kostamovaara, "Pulsed TOF laser rangefinder with a 2D SPAD-TDC receiver," IEEE Sensors, Nov 2015, DOI: 10.1109/ICSENS.2015.7370556.

[9] J. Kostamovaara, K. Määttä, M. Koskinen, R. Myllylä: Pulsed laser radars with high modulation frequency in industrial applications, Proceedings of the SPIE Conference Laser Radar VII: Advanced Technology for Applications Vol.1633, pp. 114-127, January 23-24, Los Angeles 1992.

[10] G. Bertolini, Pulse shape and time resolution. In: G. Bertolini and A Coche, Semiconductor detectors. Amsterdam, North-Holland Publishing, 1968.

[11] K. Y. Lau, Gain switching of semiconductor injection lasers. Applied physics letters, vol. 52 no.4, pp. 257-259, 1988.

[12] D. Bimberg, K. Ketterer, E. H. Bottcher, and E. Scoll, Gain modulation of unbiased semiconductor lasers: ultrashort pulse generation. Int. J. Electronics, vol. 60 no. 23, pp. 23-45, 1986.

[13] B. S. Ryvkin, E. A. Avrutin, and J. Kostamovaara, ”AsymmetricWaveguide Laser Diode for High-Power Optical Pulse Generation by Gain Switching” J. of Lightwave Technology, vol. 27, no. 12, pp. 21252131, June 2009.

[14] B. Lanz, B. Ryvkin, E. Avrutin and J. Kostamovaara, "Performance improvement by a saturable absorber in gain-switched asymmetricwaveguide laser diodes," Optics Express, 21 (24), 29780, 2013

[15] B. J. McIntyre, "Comparison of photomultipliers and avalanche photodiodes for laser applications," IEEE Trans. Electron Devices. vol. 17, no. 4, pp. 347-35, 1970.

[16] M. Hintikka, \& J. Kostamovaara, "Time domain characterization of avalanche photo detectors for sub-ns optical pulses", I2MTC, Pisa, Italy 2015 pp. 2015-2019.

[17] S.N. Vainshtein, V.S. Yuferev, and J. Kostamovaara, "Avalanche transistor operation at extreme currents: physical reasons for low residual voltages", Solid-State Electronics, vol. 47, no. 8, pp. 1255-1263, Aug. 2003.

[18] J. Nissinen and J. Kostamovaara, “A High Repetion Rate CMOS Driver for High-Energy Sub-ns Laser Pulse Generation in SPAD-Based Timeof-Flight Range Finding," IEEE Sensors Journal, vol. 6, no. 6, March 2016.

[19] F. P. Volpe, V. Gorfinkel, J. Sola \& G. Kompa, "140 W/40 ps single optical pulses for sensor application," Conference on Lasers and ElectroOptics, Anaheim, CA, USA, May 8-13, 1994.

[20] J. M. T. Huikari, E. A. Avrutin, B. S. Ryvkin, J. J. Nissinen, J. T. Kostamovaara, "High-Energy Picosecond Pulse Generation by Gain Switching in Asymmetric Waveguide Structure Multiple Quantum Well Lasers", IEEE Journal of Selected Topics in Quantum Electronics, Vol. 21, Issue 6, Article no. 1501206, 2015

[21] L. W. Hallman, J. Huikari \& J. Kostamovaara, “A high-speed/power laser transmitter for single photon imaging applications" IEEE Sensors, Valencia, Spain, 2014, pp. 1157-1160.

[22] S. A. Hovanessian, Introduction to sensor systems, Norwood MA, Artech House, 1988.

[23] T. Ruotsalainen, P. Palojärvi, T. Peltola \& J. Kostamovaara, "Laser Pulse Timing Detector" Proc. Twenty-Second European Solid-State Circuits Conference. Neuchatel, Switzerland, 1: 108-111, 1996.
[24] S. Kurtti, and J. Kostamovaara, "A wide Dynamic Range CMOS Laser Radar Receiver with a Time-Domain Walk Error Compensation Scheme," IEEE Trans. on Circ. and Syst. vol. 64, no. 3. pp. 550-561, March 2017.

[25] T. -H. Ngo, C. -H. Kim, Y. J. Kwon, J. S. Ko, D. -B. Kim and H. -H. Park, Wideband receiver for a three-dimensional ranging LADAR system, IEEE Transactions on Circuits and Systems I, vol. 60, no. 2, pp. 448-456, 2013

[26] R. J. Van de Plassche, "An 8-bit 100-MHz Fully-Nyquist Analog-toDigital Converter," IEEE J. of Solid State Circ., vol. 23, no. 6, pp. 13341344, 1988.

[27] M. L. Simpson, C. L. Britton, A. L. Wintenberg and G. R. Young, “An Integrated CMOS Time Interval Measurement System with Subnanosecond Resolution for The WA-98 Calorimeter", IEEE Journal of Solid-State circuits, vol. 32, no. 2, pp. 198-205

[28] M. Hintikka and J. Kostamovaara "A 700 MHz Laser Radar Receiver Realized in $0.18 \mu \mathrm{m}$ HV-CMOS" Springer, Analog Integrated Circuits and Signal Processing, vol. 93, no. 2, pp. 245-265, November 2017.

[29] J. Jansson, V. Koskinen, A. Mäntyniemi, and J. Kostamovaara, “A multichannel high precision CMOS time-to-digital converter for laser scanner based perception systems," IEEE trans. inst. meas., vol. 61, no. 9, pp. 2581-2590, 2012.

[30] L. Pancheri and D. Stoppa, "Low-noise CMOS single-photon avalanche diodes with 32 ns dead time," in Proc. $37^{\text {th }}$ ESSDERC, 2007, pp. 362365 .

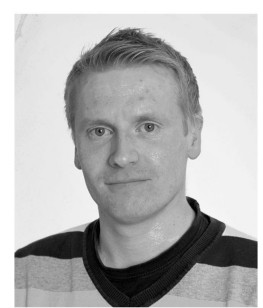

Mikko Hintikka was born in Simo, Finland, in 1983. He received an M.Sc. in electrical engineering at the University of Oulu, Finland, in 2008. After working for a few years in the mobile phone industry, he joined the Circuits and Systems research group in the University of Oulu in 2013. Since then he has been working toward doctorate in the same group. His research interests include the development of receiver channel circuits and structures for a pulsed time-of-flight laser radar devices.

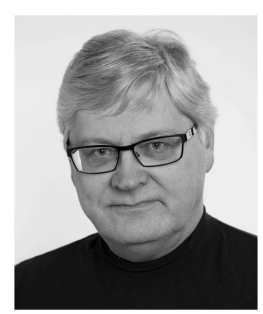

Juha Kostamovaara (M'85, S'13) received doctorate in electrical engineering from the University of Oulu, Finland, in 1987, and currently holds a full professorship in electronics at the same university. His main research interest is the development of pulsed time-of-flight devices, circuits and systems for electronic and optoelectronic measurements. 